\title{
Modeling Spatial Frailties in Survival Analysis of Cucurbit Downy Mildew Epidemics
}

\author{
P. S. Ojiambo and E. L. Kang
}

First author: Department of Plant Pathology, North Carolina State University, Raleigh 27695; and second author: Department of Mathematical Sciences, University of Cincinnati, Cincinnati, OH 45221. Accepted for publication 17 November 2012.

\section{ABSTRACT}

Ojiambo, P. S., and Kang, E. L. 2013. Modeling spatial frailties in survival analysis of cucurbit downy mildew epidemics. Phytopathology 103:216-227.

Cucurbit downy mildew caused by Pseudoperonospora cubensis is economically the most important disease of cucurbits globally, and the pathogen is disseminated aerially over a large spatial scale. Spatiotemporal spread of the disease was characterized during phase I (low and sporadic disease outbreaks) and II (rapid increase in disease outbreaks) of the epidemic using records collected from sentinel plots from 2008 to 2009 in 23 states in the eastern United States as part of the United States Department of Agriculture Cucurbit Downy Mildew ipmPIPE network. A substantive goal of this study was to explain the pattern of time to disease outbreak using important covariates while accounting for spatially correlated differences in risk of disease outbreak among the states. Survival analyses that accounts for spatial dependence were performed on time to disease outbreak, and posterior median frailties (or random effects) were mapped to identify states with high or low risk for disease outbreak. From February to October, disease occurred in 195 and 172 out of 413 and 556 cases monitored in 2008 and 2009, respectively. Disease outbreaks were spatially aggregated, with a spatial dependence of up to $\approx 1,025 \mathrm{~km}$ where clustering of outbreaks in phase I and II of the epidemic were similar. However, unlike in phase I of the epidemic, space-time point pattern analysis was significant $(P<0.0001)$ for outbreaks in phase II, during which the highest risk window as estimated by the space-time function was within 1.5 months and $500 \mathrm{~km}$ of the initial outbreak. The risk of disease outbreak peaked around July and decreased thereafter until the end of the study period. Spatially correlated analysis of time to disease outbreak indicated the need to incorporate spatial frailties in standard survival analysis models. Evaluation of alternative formulations of the spatial models demonstrated that a Bayesian hierarchical spatially structured frailty model best described time to disease outbreak. This frailty model showed clustering of outbreaks at the state level and indicated that states in the mid-Atlantic region have high spatial frailties and a high risk of downy mildew outbreak.

Additional keywords: Bayesian model, hierarchical spatial frailty model, spatial heterogeneity.
Among the diseases that affect cucurbits, downy mildew caused by Pseudoperonospora cubensis is economically the most important globally (21). The downy mildew pathogen has a high adaptive capacity that has enabled it to inhabit geographic regions with very diverse ecological conditions. Cucurbits are affected by downy mildew primarily in warm, temperate, subtropical, and tropical regions of the world. Cucumis sativus (cucumber), C. melo (cantaloupe and muskmelon), Cucurbita pepo (pumpkin and squash), and Citrullus lanatus (watermelon) are economically the most important cultivated hosts affected by the disease (21). The disease spreads rapidly when environmental conditions are favorable and can destroy the entire host within 4 to 7 days. Severe epidemics result in a significant reduction in yield quality and quantity (21). In 2004, severe epidemics of the disease occurred in the eastern United States that devastated the cucumber crop, leading to substantial yield losses (18). There are no commercial varieties that are resistant to downy mildew, and disease control relies primarily on the use of fungicides. Thus, monitoring the occurrence and dissemination of $P$. cubensis is a key element in the management of cucurbit downy mildew (25).

Cucurbits are very sensitive to frost and $P$. cubensis can only survive in frost-free areas in southern Florida and along the Gulf of Mexico in the United States. Thus, epidemics of downy mildew in the continental United States depend on the annual dispersal of sporangia from the subtropical overwintering sources in the south.

Corresponding author: P. S. Ojiambo; E-mail address: peter_ojiambo@ncsu.edu

http://dx.doi.org/10.1094/PHYTO-07-12-0152-R

(c) 2013 The American Phytopathological Society
In this regard, disease outbreaks in overwintering areas in the south can serve as a baseline for estimating the time to disease outbreak in the continental United States (2). Although the disease occurs annually in the eastern United States, its spread from overwintering areas to fields in the northern states is the most uncertain feature within the framework of epidemic prediction (25). This uncertainty is expected because regional spread of disease tends to be curtailed by limited opportunities for pathogen establishment than by atmospheric transport of inoculum (2). Thus, understanding the processes generating observed patterns of disease distribution can enable reliable prediction of the spread and, hence, adoption of appropriate measures to contain the disease. Sporangia of $P$. cubensis can be disseminated aerially over large spatial scales. Recently, the spatiotemporal spread of the disease in the eastern United States was characterized and it has been proposed that infection of cucurbits by $P$. cubensis appears to be an outcome of a contagion process and that the space-time infection risk window is relatively large (24). However, changes in the spatiotemporal interaction of the infection risk during the growing season have not been analyzed. Further, the risk of disease outbreak in the eastern United States and associated risk factors has not been determined. Understanding the dynamics of spatiotemporal interaction and establishing the risk of disease outbreak implies that decisions concerning practical issues such as disease monitoring periods can be made with greater objectivity.

When an outcome of interest is time to disease outbreak in a field, standard time-to-event (i.e., survival or failure time) models (13) can be used to estimate the risk of disease outbreak $(26,31)$. Standard time-to-event time models assume that observations of 
interest are statistically independent after controlling for available risk factors (1). However, the assumption of independence presents two concerns with regard to risk estimation. First, if the assumption of statistical independence is not valid, the uncertainty in the risk estimates of the event of interest (e.g., disease outbreak) may be stated incorrectly. Second, even after controlling for available risk factors, time to disease outbreak in fields closer together may be more similar than in fields that are farther apart, which results in spatial autocorrelation. If this spatial autocorrelation is due to missing or systematically incorrect estimates of risk factors that are spatially correlated with disease outbreak, then estimates of the risk of disease outbreak may be biased due to inadequate control of these factors. A possible solution to this problem is to incorporate a random effect (usually termed as a frailty) in time-to-event models within a spatial framework to account for unobserved heterogeneity in the event time. The spatial conditional autoregressive (CAR) model (8) is typically the model of choice for describing spatial dependence between such random effects at different locations. In the CAR model, the conditional mean of the frailty in a given area is a weighted average of the frailties in neighboring areas, while the conditional variance is inversely proportional to the sum of the neighbor weights.

In statistical terms, a frailty model is a random effects model for time-to-event data where the frailty has a multiplicative effect on the baseline hazard function (19). In this article, we apply a Bayesian hierarchical frailty model to downy mildew outbreaks in the eastern United States and present a Bayesian time-to-event model with spatially correlated frailties following the CAR model. Due to the complexity of the Bayesian hierarchical statistical models, it is not feasible to directly compute the posterior distributions. Instead, simulation procedures such as the Markov chain Monte Carlo (MCMC) sampling (16) are used to generate empirical estimates of the posterior distribution. Bayesian hierarchical frailty models have been applied in medical epidemiology and biostatistics $(5,15)$, where the resulting posterior medians of frailties are used to identify spatial or spatiotemporal trends. The effect of covariates in explaining the observed frailty patterns can also be assessed using these models. Although spatial heterogeneity can impact outbreaks of plant diseases substantially (27), there have been no attempts to incorporate frailties in time-to-event analysis in botanical epidemiology. Thus, the main objectives of this study were to (i) describe the spatiotemporal interaction of infection risk and describe changes in the component of this interaction that occurred over the course of two distinct phases of the downy mildew epidemic and (ii) apply standard time-to-event models to downy mildew outbreaks in the eastern United States to model subject-specific fixed effects due to various covariates and unobserved heterogeneity due to frailties whose spatial correlation is described through the CAR model.

\section{THEORY AND APPROACHES}

Database for disease records. Disease records used in this study were collected in 2008 and 2009 as part of the Cucurbit Downy Mildew (CDM) ipmPIPE project (25). The CDM ipmPIPE is a disease-monitoring network that is part of the United States Department of Agriculture Pest Information Platform for Extension and Education (PIPE) program. Records of the chronology of confirmed disease outbreaks were collected from the CDM ipmPIPE sentinel plot network. Sentinel plots were fixed plots, planted early in the season and designated a priori for surveillance at least once a week. Five cucurbit host types (namely, cantaloupe, cucumber, pumpkin, squash, and watermelon) were planted in each sentinel plot. Collaborators within the network scouted plots regularly and reported new outbreaks online via the project's website (http://cdm.ipmpipe.org/). Disease records used in this study were obtained from 23 states in the eastern United States. These states were selected for detailed analyses because they appeared to form a contiguous epidemic unit based on the general pattern of disease progress (24). The following variables were used in subsequent analyses: (i) date of disease outbreak, (ii) state, (iii) host type affected, and (iv) latitude and longitude for each sentinel plot.

Spatial analysis. To describe the spatial distribution and spatiotemporal interaction among disease outbreaks, two epidemic phases that represent the natural temporal progress of downy mildew were defined: phase I (a period of low and sporadic disease outbreaks) and phase II (a period of rapid increase in disease outbreaks). An epidemic is usually considered to have entered phase II at the time when $5 \%$ of the cumulative disease outbreaks are observed (36). This time period occurred during the first week of June and, thus, phase I was defined as the period from February to May while phase II was the time period from June to September.

The $O$-ring statistic, $O(s)$, was used to characterize the spatial pattern of disease outbreaks in phases I and II. The $O(s)$ is the intensity of points of a given point pattern within a ring around an arbitrary point of a reference pattern (38). The $O(s)$ was calculated using the software PROGRAMITA, and analytical details and implementation of the $O(s)$ are presented elsewhere (38). Monte Carlo simulations under a specific null model of complete spatial randomness were used to construct confidence intervals for the $O(s)$ statistic for the observed pattern. In total, $m /(m+1) \times 100 \%$ confidence interval bands were calculated individually from the highest and lowest values of the $m$ test statistics based on $m$ simulations of the null model. Here, 199 simulations were conducted (i.e., $m=199$ ), which corresponds to a $99.5 \%$ confidence interval bands. A statistic was judged as an indication for a departure from the null model at that spatial scale if it was outside the confidence interval at a given distance.

Spatiotemporal analysis. The extended $K$ function in spacetime patterns was used to test for space-time interactions in the risk of infection in phases I and II of the epidemic. The $K$ functions (28) have been commonly used to characterize whether events such as disease outbreaks are completely randomly distributed in space or time and whether there is spatial or spatiotemporal dependence. For example, if there is clustering, we expect to see more outbreaks within a short distance in space and time, compared with a process with completely spatiotemporal randomness.

Briefly, if $K(s)$ and $K(t)$ and denote the $K$ function in space and time, respectively, the $K$ function difference $D(s, t)$ is computed as (14)

$$
D(s, t)=K(s)-[K(s) \cdot K(t)]
$$

If disease outbreak patterns in space and time are independent (i.e., no space-time interaction), the space-time $K$ function $K(s, t)$ is expected to be close to the product of separate $K$ functions. Thus, departures of $D(s, t)$ from zero indicate presence of spacetime interaction. To empirically compare space-time interactions among time periods and spatial regions, we standardized the difference $D(s, t)$ by the associated spatial and temporal intensity and computed $D_{0}(s, t)$ as

$$
D_{0}(s, t)=D(s, t) /[K(s) \cdot K(t)]
$$

The SPLANCS library in R was used to conduct formal tests for the presence of space-time interaction in the patterns of disease outbreaks. Specifically, we generated $m$ Monte Carlo simulations in which each of the $n$ case events were labeled with the observed $n$ time "markers". In total, $m$ estimates of $D(s, t)$ were computed and the sum of $D(s, t)$ over all $s$ and $t$ was calculated for each simulation. The sum of $D(s, t)$ for the observed data was then ranked among the empirical frequency of these $m$ sums. If the 
observed sum ranked $k$ th largest (or smallest), the one-sided attainedsignificance level was $\mathrm{k} / \mathrm{m}$. Space-time interaction is established to be present if the following hold: (i) peaks on the surface of $D_{0}(s, t)$ plotted over $s$ and $t$, (ii) values of standardized residuals $>2$ units above or below the expected value of zero, and (iii) a significant test statistic from the Monte Carlo simulations (24).

Standard survival analysis of time to disease outbreak. In this study, the event of interest was downy mildew outbreak and time-to-event was expressed as time (days) to disease outbreak after the first disease report in southern Florida. The first disease outbreak in southern Florida was reported on 18 February and 23 March 2008 and 2009, respectively. In the first set of analysis, two categorical variables were constructed: (i) host type and (ii) regional location of disease outbreak. Host types were classified into three groups: cucumber, squash, and other cucurbit species. Other species included watermelon, cantaloupe, and pumpkin. Outbreaks were also grouped into two regions depending on whether the outbreaks occurred in the southern- or northern-tier states. Distribution functions for time to disease outbreak were obtained using the Kaplan-Meier estimation within the LIFETEST procedure in SAS (1) and differences between groups within each categorical variable were compared using the logrank test. Kaplan-Meier product limit estimates of the weekly risk of disease outbreak (i.e., hazard) following the first outbreak in southern Florida were also calculated and plotted to visualize patterns of the risk of disease outbreak over time.

Bayesian spatial frailty modeling of time to disease outbreak. Within the Bayesian framework, prior beliefs about the quantities of interest and information from observations (i.e., current data) are combined to make inference on unknown quantities of interest (e.g., parameters or predictions). Let $\boldsymbol{\theta}$ be a set (i.e., a vector) of unknown parameters and $\mathbf{Z}$ be the observed data. Further, suppose that $P(\mathbf{Z} \mid \boldsymbol{\theta})$ is the likelihood function, and $P(\boldsymbol{\theta})$ is the prior probability of $\boldsymbol{\theta}$ based on some prior knowledge or assumptions (but no information from data). In the Bayesian framework, $\boldsymbol{\theta}$ is estimated using the Bayes' rule as

$$
P(\boldsymbol{\theta} \mid \mathbf{Z})=[P(\boldsymbol{\theta}) \cdot P(\mathbf{Z} \mid \boldsymbol{\theta})] /[P(\mathbf{Z})] \propto P(\boldsymbol{\theta}) \cdot P(\mathbf{Z} \mid \boldsymbol{\theta})
$$

where $P(\bullet)$ is a probability and $P(\bullet \mid \bullet)$ is a conditional probability. The posterior probability, $P(\boldsymbol{\theta} \mathbf{Z})$ is determined from equation 3 using $P(\boldsymbol{\theta})$ and $P(\mathbf{Z} \mid \boldsymbol{\theta})$. Inference on the unknown $\boldsymbol{\theta}$ can then be made using the posterior distribution $P(\boldsymbol{\theta} \mathbf{Z})$. For example, the posterior median is typically used as a point estimator of $\boldsymbol{\theta}$.

Given that parameters and unobserved (latent) random processes or vectors need to be estimated in most statistical models, it is convenient to build Bayesian hierarchical statistical models (39) using a series of hierarchical steps: (i) data model, $P(\mathbf{Z} \mid \mathbf{Y}, \boldsymbol{\theta})$; (ii) process model, $P(\mathbf{Y} \mid \boldsymbol{\theta})$; and (iii) parameter model (or prior), $P(\boldsymbol{\theta})$; where $\mathbf{Z} \equiv$ data, $\mathbf{Y} \equiv$ process, and both $\mathbf{Y}$ and $\boldsymbol{\theta}$ are the unknowns. The parameter $\boldsymbol{\theta}$ is assumed to have a prior probability distribution associated with the parameter model. The posterior distribution of the unknowns is then calculated using the equation

$$
P(\mathbf{Y}, \boldsymbol{\theta} \mathbf{Z}) \propto P(\mathbf{Z} \mid \mathbf{Y}) \cdot P(\mathbf{Y} \mid \boldsymbol{\theta}) \cdot P(\boldsymbol{\theta})
$$

based on which inferences on $\mathbf{Y}$ and $\boldsymbol{\theta}$ can be made.

We adopted this three-step hierarchical approach to model subject-specific fixed effects due to covariates and unobserved heterogeneity due to frailties. Let $t_{i j}$ be the time to disease outbreak or censoring for the host field $j$ and in the state (i.e., stratum) $i$, and let $\gamma_{i j}$ be the corresponding disease indicator ( 0 for a noninfected and 1 for an infected host field), for $j=1, \ldots, n_{i}$ and $i=1, \ldots, I$. Further, let $\mathbf{t}=\left\{t_{i j}\right\}$ be a vector of observed times to disease outbreak and $\gamma=\left\{\gamma_{i j}\right\}$ denote the vector of corresponding disease indicators. Starting with the data model, we consider the probability distribution of the observed data $(\mathbf{t}, \gamma)$ conditional on the processes and parameters. First, we assume proportional haz$\operatorname{ards} \mathrm{h}\left(t_{i j} ; \mathbf{x}_{i j}\right)$ (12), which leads to a model of the following form

$$
h\left(t_{i j} ; \mathbf{x}_{i j}\right)=h_{0}\left(t_{i j}\right) \exp \left(\boldsymbol{\beta}^{T} \mathbf{x}_{i j}\right)
$$

where $h_{0}\left(t_{i j}\right)$ is the baseline hazard, $\mathbf{x}_{i j}$ is a $p$-dimensional vector of individual-specific covariates, and $\boldsymbol{\beta}$ is the corresponding vector of coefficients. Specifically, $\boldsymbol{\beta}^{T} \mathbf{x}_{i j}=\left\{\beta_{0}, \beta_{1} \mathbf{x}_{i j}\right\}$, in which $\beta_{0}$ is an intercept term $(9,35)$. An intercept term is usually not explicitly estimated in a proportional hazards model. However, inclusion of an intercept term dramatically improves the convergence of the procedure (35). Thus, the inclusion of $\beta_{0}$ in equation 5 is purely a computational issue because it makes no difference in the final model because the exponentiated intercept term will simply be absorbed into the unknown $h_{0}\left(t_{i j}\right)$. After careful exploratory data analysis, three covariates were defined in this study: (i) the intercept, $\beta_{0}$ (a single intercept for all host fields), (ii) an indicator variable for the host group cucumber (host $=1$ ), and an indicator variable for the host group squash (host $=2$ ). Thus, the reference group in the analysis was other cucurbit species (host $=3$ ).

To incorporate a frailty (or random effect) term, equation 5 is extended to

$$
h\left(t_{i j} ; \mathbf{x}_{i j}\right)=h_{0}\left(t_{i j}\right) \omega_{i} \exp \left(\boldsymbol{\beta}^{T} \mathbf{x}_{i j}\right)=h_{0}\left(t_{i j}\right) \exp \left(\boldsymbol{\beta}^{T} \mathbf{x}_{i j}+W_{i}\right)
$$

where $W_{i} \equiv \log \omega_{\mathrm{i}}$ is the state-specific frailty term representing unobserved heterogeneity among different clusters. In this study, all host fields in the same state composed a single cluster. These frailties, $\left\{W_{i}\right\}$, are random effects and will be described below in the second hierarchical step, the process model. Although a variety of models have been used to describe the parametric form for the baseline hazard $h_{0}\left(t_{i j}\right)$, we adopted the Weibull model that represents a good tradeoff between simplicity and flexibility (6). Applying the Weibull model to describe the baseline hazard in equation 6 results in a hazard rate of the form

$$
h\left(t_{i j} ; \mathbf{x}_{i j}\right)=\rho t_{i j}^{\rho-1} \exp \left(\boldsymbol{\beta}^{T} \mathbf{x}_{i j}+W_{i}\right)
$$

where $\rho$ is the Weibull shape parameter. Values of $\rho>1$ reflect a monotonically increasing hazard, while $\rho<1$ reflects a monotonically decreasing hazard (1). The median time to disease outbreak (i.e., time after which $50 \%$ of the host fields have become infected), $m_{i j}$, is derived from equation 7 and was estimated based on a modified equation of Spiegelhalter et al. (33) as

$$
m_{i j}=\left[\ln 2 e^{-\left(\boldsymbol{\beta} \mathbf{x}_{i j}+\mathrm{W}_{i}\right)}\right]^{1 / \rho}
$$

Thus, the data model for $(\mathbf{t}, \gamma)$ given the process $\mathbf{W}=\left\{W_{\mathrm{i}}\right\}$ and parameters $\{\boldsymbol{\beta}, \rho\}$ was expressed as follows:

$$
P(\boldsymbol{\beta}, \mathbf{W}, \rho \mid \mathbf{t}, \boldsymbol{\gamma}) \propto \prod_{i=1}^{I} \prod_{j=1}^{n_{i}}\left\{\rho t_{i j}^{\rho-1} \exp \left(\boldsymbol{\beta}^{T} \mathbf{x}_{i j}+W_{i}\right)\right\}^{\gamma_{i j}} \exp \left\{-t_{i j}^{\rho} \exp \left(\boldsymbol{\beta}^{T} \mathbf{x}_{i j}+W_{i}\right)\right\}
$$

Time to disease outbreak in the field can be easily grouped into states and, in the second stage of the hierarchical model, the process model, we described the unknown state-specific frailties, $\mathbf{W}=\left\{W_{i}\right\}$. These state-specific frailties were considered to be either spatially unstructured or spatially structured. In the spatially unstructured setting, $\mathbf{W}$ in the process model was assumed to be distributed as follows:

$$
W_{i}^{i i d} \sim N\left(0, \sigma^{2}\right)
$$

For the spatially structured setting, spatial dependence between frailty terms was specified by their conditional distribution as

$$
W_{i} \mid W_{i^{\prime} \neq i} \sim N\left(\alpha_{i}, \sigma_{i}^{2}\right)
$$


with the conditional mean $\alpha_{i}$ being given as $\alpha_{i}=\sum_{i^{\prime}=1}^{I} \phi_{i i^{\prime}} W_{i^{\prime}} / \sum_{i^{\prime}=1}^{I} \phi_{i i^{\prime}}$ and the conditional variance $\sigma_{i}^{2}$ of the form: $\sigma_{i}^{2}=\sigma^{2} / \sum_{i^{\prime}=1}^{I} \phi_{i i^{\prime}}$ where $\phi_{i i^{\prime}}$ denotes the neighborhood weights. The neighborhood weights were binary, so that $\phi_{i i^{\prime}}=1$ for states $i$ and $i^{\prime}$ for adjacent state or states with a common boundary, and $\phi_{i i^{\prime}}=0$ otherwise. This is equivalent to assuming a process model of the following structure:

$$
\mathbf{W} \mid \lambda \sim \operatorname{CAR}(\lambda)
$$

where $\lambda\left(=1 / \sigma^{2}\right)$ is intensity of the spatial process and CAR represents a spatial conditional autoregressive structure (8). It has been shown (7) that conditional on the parameter $\lambda, \mathbf{W}$ has the probability distribution proportional to

$$
\lambda^{1 / 2} \exp \left[-\frac{\lambda}{2} \sum_{i \text { adj } i^{\prime}}\left(W_{i}-W_{i^{\prime}}\right)^{2}\right] \propto \lambda^{1 / 2} \exp \left[-\frac{\lambda}{2} \sum_{i=1}^{I} m_{i} W_{i}\left(W_{i}-\bar{W}_{i}\right)\right]
$$

where $i$ adji' denotes that the states $i$ and $i^{\prime}$ are adjacent, $\bar{W}_{i}$ is the mean of the $W_{i \neq i}$ that are adjacent to $W_{i}$, and $m_{i}$ is the number of these adjacencies. In order to allow the data to identify an intercept term $\beta_{0}$ in the hazard function in equation 6 , the constraint $\sum_{i^{\prime}=1}^{I} W_{i}=0$ was added to the model specification as suggested by Banerjee et al. (6). A consequence of this prior specification for the process $\mathbf{W}=\left\{W_{i}\right\}$ is that

$$
W_{i} \mid W_{i^{\prime}=i} \sim N\left[\bar{W}_{i}, 1 /\left(\lambda m_{i}\right)\right]
$$

To complete the Bayesian hierarchical model, we specify the parameter model (prior distribution) for the following: vector of coefficients, $\boldsymbol{\beta}$; the Weibull parameter $\rho$, and $\lambda$ from the CAR model in the spatially structured frailty setting (or the parameter $\sigma^{2}$ within the spatially unstructured setting). Given that we did not have prior knowledge about the parameters, we assumed model parameters to be independent with noninformative priors (20). Typically, noninformative prior specifications in Bayesian disease mapping are assumed for the parameters to allow the data to play the principal role in determining the posterior distribution of the parameters (20). Thus, we assumed a classic flat (improper uniform) prior (i.e., between zero and infinity) for $\beta$, and assumed a vague but proper $\gamma$ distribution prior, $\mathrm{G}(\alpha, 1 / \alpha)$ with $\alpha=10^{-4}$ (mean 1 , variance $10^{4}$ ), for both $\rho$ and $\lambda$ (or $1 / \sigma^{2}$ in the spatially unstructured setting).

Implementation of the Bayesian hierarchical spatial models. The models outlined above with unstructured and spatial structured frailty terms were implemented in a Bayesian framework using the MCMC (16) methods in WinBUGS (33). The joint posterior distribution of the unknowns (i.e., frailties and parameters) given the observed data $(\mathbf{t}, \boldsymbol{\gamma})$ was expressed as

$$
P(\boldsymbol{\beta}, \mathbf{W}, \rho, \lambda \mid \mathbf{t}, \mathbf{x}, \boldsymbol{\gamma}) \propto L(\boldsymbol{\beta}, \mathbf{W}, \rho ; \mathbf{t}, \mathbf{x}, \boldsymbol{\gamma}) P(\mathbf{W} \mid \lambda) P(\boldsymbol{\beta}) P(\rho) P(\lambda)
$$

where the first term on the right-hand side is the Weibull likelihood in equation 8 , the second term is the CAR distribution of the random frailties, and the rest of the terms are prior distributions. The updated Weibull likelihood from equation 9 is then be expressed as

$$
L(\boldsymbol{\beta}, \mathbf{W}, \rho ; \mathbf{t}, \mathbf{x}, \boldsymbol{\gamma}) \propto \prod_{i=1}^{I} \prod_{j=1}^{n_{i}}\left\{\rho t_{i j}^{\rho-1} \exp \left(\boldsymbol{\beta}^{T} \mathbf{x}_{i j}+W_{i}\right)\right\}^{\gamma_{i j}} \exp \left\{-t_{i j}^{\rho} \exp \left(\boldsymbol{\beta}^{T} \mathbf{x}_{i j}+W_{i}\right)\right\}
$$

Metropolis random walk steps with Gaussian proposals were used for sampling from the full conditionals for $\boldsymbol{\beta}$, while Hastings independence steps with gamma proposals were used for updating $\rho$ (10). Posterior median frailties were monitored and used for mapping the results by state. For both the unstructured and spatially structured models, five parallel chains were run with different starting values; the burn-in period was 50,000 iterations, and the estimation sample comprised $5 \times 10,000=50,000$ values after a thinning of the chains by a factor of 10 . Convergence of the models was assessed using correlation and sample trace plots.

Bayesian model choice and checking. The use of a flat prior for $\boldsymbol{\beta}$ precludes the use of Bayes factors for model choice and instead we turned to the deviance information criterion $(D I C)$, an extension of the Akaike information criterion (AIC) for hierarchical models. The DIC is based on the posterior distribution of the deviance statistic:

$$
D(\boldsymbol{\theta})=-2 \log f(\mathbf{Z} \mid \boldsymbol{\theta})+2 \log h(\mathbf{Z})
$$

where $f(\mathbf{Z} \mid \theta)$ is the likelihood function for the observed data vector $\mathbf{Z}$ given the parameter vector $\boldsymbol{\theta}$, and $h(\mathbf{Z})$ is some standardizing function of the data alone. Using this approach, the fit of a model is summarized by the posterior expectation of the deviance, $\bar{D}=\mathrm{E}_{\mathrm{g} Z}[D]$, while the complexity of a model is captured by the effective number of parameters, $p_{D}$. Spiegelhalter et al. (32) show that $p_{D}$ can reasonably be defined as

$$
p_{D}=\mathbf{E}_{\theta \mathrm{Z}}[D]-D\left(E_{\theta \mathrm{Z}}[\boldsymbol{\theta}]=\bar{D}-D(\overline{\boldsymbol{\theta}})\right.
$$

(i.e., the expected deviance minus the deviance evaluated at the posterior expectations). Due to the borrowing strength across frailties, $p_{D}$ will be less than the actual total number of parameters in the model. The DIC is then defined as the expected deviance plus the effective number of parameters (i.e., $D I C=\bar{D}+p_{D}$. Because small values of $\bar{D}$ and $p_{D}$ indicate a better fit and a parsimonious model, respectively, small values of the sum (DIC) indicate preferred models. However, the DIC is not intended for identification of the "correct" model but, rather, a method of comparing a collection of alternative formulations. Because DIC is scale free, the choice of standardizing function $h(\mathbf{Z})$ in equation 16 is arbitrary. Thus, values of $D I C$ have no intrinsic meaning and only the differences in DIC across models are meaningful. As a rule of thumb, models receiving DIC within 1 to 2 units of the "best" model deserve consideration, whereas those with DIC within 3 to 7 units of the best model have considerably less support (32). The distribution of the posterior median frailties were also examined using box-whisker plots to check the quality of the competing models (6).

\section{RESULTS}

Seasonal development of disease epidemics. Downy mildew outbreaks displayed a clear trend during the course of the disease epidemic in both years. This was characterized by low and sporadic outbreaks at the start of the epidemic, followed by a rapid increase in the new outbreaks and then a gradual decrease in new outbreaks toward the end of the epidemic (Fig. 1). New outbreaks peaked $\approx 1.5$ months earlier in 2009 (end of June) compared with the 2008 epidemic (early August). Of the 413 fields monitored in 2008, 195 fields were infected, whereas 172 fields were infected of 556 fields monitored in 2009 (Table 1). A higher proportion of disease outbreaks was reported on cucumber in $2008(40.5 \%)$ than in $2009(26.2 \%)$ compared with all other cucurbit host types.

Spatial and spatiotemporal aggregation of disease cases. In both years, disease outbreaks showed a more significant aggregation $(P<0.001)$ than the distribution expected from the null hypothesis of complete spatial randomness in phase I and II of the epidemic. However, the extent of spatial clustering in phase I was not very different from that observed in phase II of the epidemic. For example, the spatial extent was $\approx 975$ and $925 \mathrm{~km}$ in phase I and II, respectively, in 2008. Similar results were also observed in 2009 , where the extent of spatial clustering was 1,125 and $1,075 \mathrm{~km}$ in phase I and II, respectively. 
Unlike spatial clustering, there were consistent differences in the extent and significance of space-time interaction between the two phases of the epidemic. In 2008, $D_{0}(s, t)$ ranged from -0.3 to 5.2 in phase I and -0.2 to 6.3 in phase II of the epidemic. The $D_{0}(s, t)$ plots indicated defined peaks at a distance of $\approx 450 \mathrm{~km}$ and 2 months in phase I and a distance of $500 \mathrm{~km}$ (Fig. 2A) and 1 month in phase II (Fig. 2B) in 2008. However, the Monte Carlo simulations were significant $(P=0.001)$ only for the observed space-time pattern in phase II (Fig. 2C and D). In 2009, $D_{0}(s, t)$ ranged from -0.1 to 1.7 in phase I and -0.1 to 5.1 in phase II and the $D_{0}(s, t)$ plots had defined peaks at a distance of $\approx 500 \mathrm{~km}$ and 1.5 months in phase I and a distance of $400 \mathrm{~km}$ and 1 month in phase II of the epidemic. Similarly, simulation tests in 2009 were significant $(P=0.001)$ only for the observed space-time pattern in phase II of the epidemic (data not shown).

Disease risk and standard survival analysis of time to disease outbreak. Time to disease outbreak varied considerably across the 23 states in both years (Fig. 3). The weekly risk of disease outbreak was generally low and static during phase I but rapidly increased during phase II of the epidemic (Fig. 4). In 2008, the risk peaked during the week of 18 July (Fig. 4A), whereas the risk peaked in the week starting 1 May in 2009 (Fig. 4B). The weekly risk of disease outbreak at the peak period in 2008 was $\approx 25 \%$ higher than that in 2009 . In both years, the risk of disease outbreak gradually declined toward the end of the epi- demic after the peak period (Fig. 4). The weekly risk of disease outbreak differed between cucurbit host types, especially during phase II of the epidemic (Fig. 4). In 2008, the weekly risk increased rapidly for cucumber compared with squash or other cucurbit host types. At the peak period, the risk was three to five times higher for cucumber compared with other hosts (Fig. 4C). Similar results were also observed in 2009, except that the risk of disease outbreak was two to three times higher for squash compared with cucumber or other cucurbit species at the peak period (Fig. 4D).

Cucurbit host type significantly $(P<0.0001)$ affected time to disease outbreak in both years. In 2008 , time to disease outbreak was shorter in cucumber than in squash, whereas the converse was observed in 2009. The effect of the region on time to disease outbreak was only significant $(P<0.0001)$ in 2008, where disease outbreaks occurred $\approx 2$ weeks earlier in southern- than northerntier states (Table 2).

Bayesian comparison of competing spatial models. The effective number of parameters, $p_{D}$, for the model with no frailty was 4.07 and 3.98 in 2008 and 2009, respectively (Table 3). These values were approximately equal to the actual number of parameters in the model, which was four (three components of $\boldsymbol{\beta}$ and the Weibull parameter $\rho$ ). In comparison, values of $p_{D}$ for the unstructured and spatially structured frailty models were substantially larger but much smaller than their actual parameter counts (which

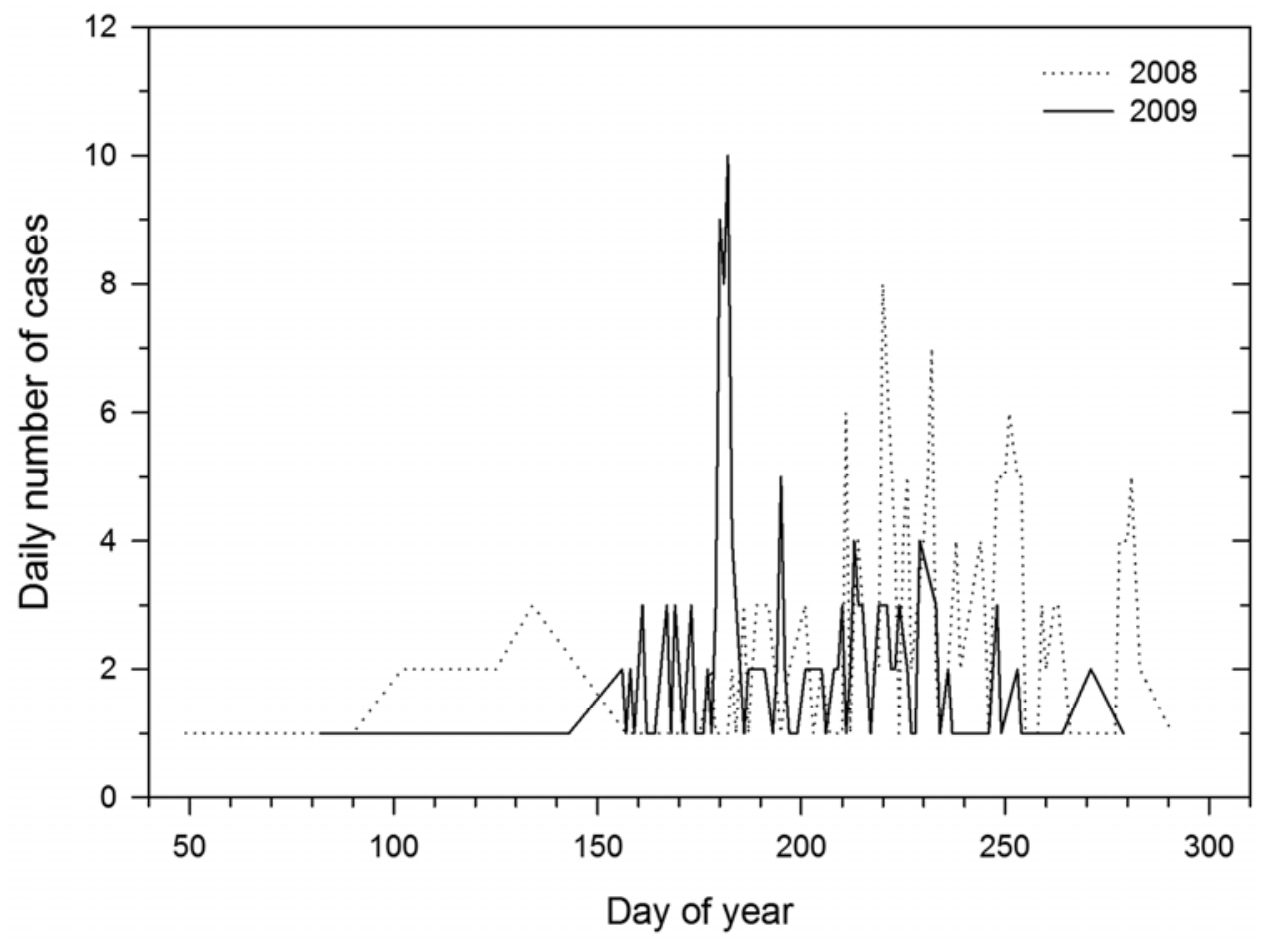

Fig. 1. Temporal increase in the daily number of new outbreaks of cucurbit downy mildew (caused by Pseudoperonospora cubensis) in disease monitoring field plots in the eastern United States during the growing season in 2008 and 2009.

TABLE 1. Counts of infected and noninfected cases for different cucurbit host types reported in disease monitoring plots during epidemics of cucurbit downy mildew in the eastern United States in 2008 and 2009

\begin{tabular}{|c|c|c|c|c|c|c|}
\hline \multirow[b]{3}{*}{ Host type } & \multicolumn{6}{|c|}{ Number of cases } \\
\hline & \multicolumn{2}{|c|}{2008} & \multicolumn{2}{|c|}{2009} & \multicolumn{2}{|c|}{ Total $^{\mathrm{a}}$} \\
\hline & Infected & Noninfected & Infected & Noninfected & 2008 & 2009 \\
\hline Squash & 76 & 42 & 79 & 80 & 118 & 159 \\
\hline Others ${ }^{b}$ & 40 & 137 & 48 & 191 & 177 & 239 \\
\hline Total & 195 & 218 & 172 & 384 & 413 & 556 \\
\hline
\end{tabular}

a Total number of sentinel plots for different cucurbit host types monitored for cucurbit downy mildew in respective years.

b Other host types include cantaloupe, pumpkin, and watermelon. 
would include 23 random frailties). A smaller value of $p_{D}$ relative to the actual number of parameters in the model reflects a parsimonious model. Despite their increased size, the two frailty models had smaller DIC values compared with the model with no frailty (Table 3). Further, the model with spatially structured frailty terms had the smallest DIC (considered the best model) compared with either the model with unstructured frailty terms (second-best model) or with no frailty term. Using the spatially structured model as the base model, $\triangle D I C$ value for the unstructured frailty model was $\approx 7.0$ units (Table 3 ), indicating that time to disease outbreak was best described by the spatially structured frailty model. This observation is also supported by the distribution of the posterior median frailties, where the tightness of the boxplots for the structured frailty model (Fig. 5) suggested that the model was best at reducing the need for the frailty terms and reducing lack-of-fit in the spatial model.

Spatially structured survival analysis. The estimated median time to disease outbreak was 203 to 242 days in 2008 and 157 to 243 days in 2009. In both years, cucumber (host $=1$ ) had a higher risk of disease outbreak compared with other cucurbit species (reference host group, host $=3$ ). The posterior median risk of disease outbreak for cucumber increased by a factor of $e^{0.778}=$ 2.18 in 2008 and $e^{0.434}=1.54$ in 2009 compared with other cucurbit species (Table 4). Similarly, compared with other cucurbit species, the risk of disease outbreak on squash (host $=2$ ) decreased by a factor of 0.76 in 2008 but increased dramatically by a factor of 3.32 in 2009. In addition, the estimate of the Weibull parameter $\rho$ was higher in $2008(\rho=3.604)$ than in $2009(\rho=$ 1.970) (Table 4), indicating a faster increase in the baseline risk of disease outbreak in 2008 than in 2009. In addition, estimates of $\rho$ were $>1$, indicating that baseline risk of disease outbreak in both years was increasing over time during the epidemic.

Choropleth maps of the posterior 2.5th, 50th (median), and 97.5th percentiles for the frailty terms $W_{i}$ from the model with spatially structured frailty terms in 2008 and 2009 are presented in Figure 6. Across the 2 years of study, the range of the posterior medians was -0.8 to 0.8 . The corresponding posterior standard deviations across the 2 years of study were much smaller, 0.03 to 0.08 . Choropleth maps of the posterior 2.5th, 50th, and 97.5th percentiles based on an interval of 0.2 had a similar clustering pattern of the posterior frailties (Fig. 6), indicating that the 0.2 classification interval resulted in a significant difference in distribution of the frailties between states in the region. Positive posterior frailty values indicate an increase in the risk of disease outbreak, whereas negative posterior frailty values indicate a decreasing risk of disease outbreak. Thus, the states of North Carolina and Virginia had higher median frailties and, therefore, higher risk of downy mildew outbreaks than all other states in the eastern United States in both years (Fig. 6). In addition, Georgia had a much higher median frailty and risk of downy mildew outbreaks in 2008 than in 2009. We also observed clusters of states with lower median frailties and lower risks of disease outbreak in the southwest part of the United States and around the Great Lakes in 2008 and in the southern states in 2009 (Fig. 6).
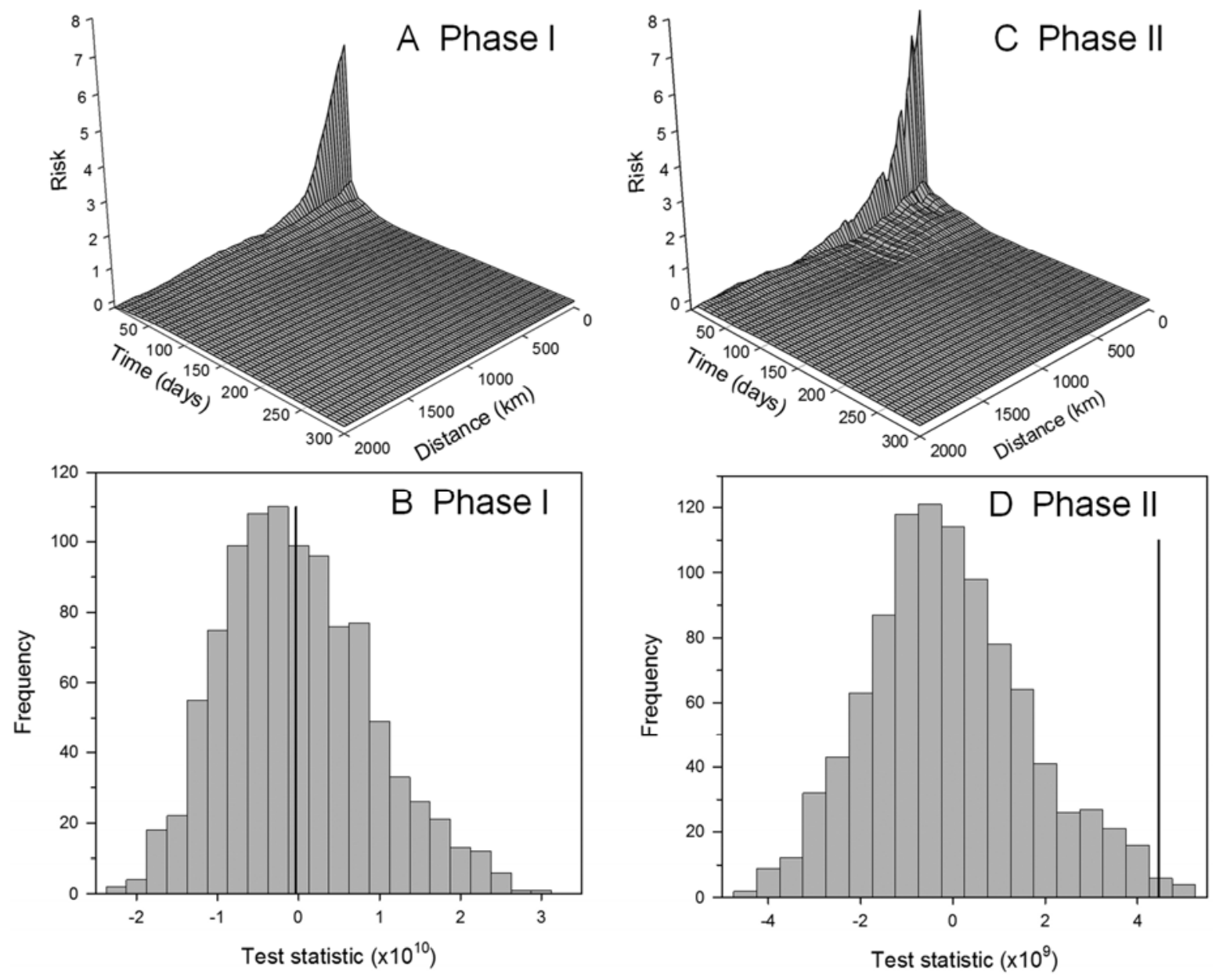

Fig. 2. Spatiotemporal interaction $\left[D_{0}(s, t)\right]$ plots and related statistics for a point-pattern analysis of cucurbit downy mildew risk among sentinel plots in the eastern United States in 2008. A, Space-time interaction for disease outbreaks reported during phase I of the epidemic and B, the corresponding test statistics from Monte Carlo simulations. C, Space-time interaction for outbreaks reported in phase II of the epidemic and D, the corresponding test statistics from Monte Carlo simulations. Evidence of space-time interaction is indicated by positive peaks in the value of $D_{0}(s, t)$ that is also above the 95 th quartile value for that time and distance for 999 simulations. The vertical thick line in the Monte Carlo simulations (lower panels) is the test statistic calculated from the observed data. 
Overall, states in the mid-Atlantic region had high median frailties and a higher risk of disease outbreak during the downy mildew epidemic in 2008 and 2009.

\section{DISCUSSION}

This study examined cucurbit downy mildew epidemics in the eastern United States to gain insight into changes in the spatial and spatiotemporal interaction of the infection risk that occur during different phases of the epidemic. Further, we extended standard survival analysis models to a spatial setting to account for unobserved spatially correlated heterogeneity in time to disease outbreak to better estimate the risk of disease outbreak with- in the region. The database used in this study is based on a dense network of fixed-site plots that are monitored weekly, and this allows for an accurate assessment of time to disease outbreak. Our results indicate that clustering of disease outbreaks does not change significantly during the season but the space-time risk window varies significantly during the course of the epidemic. Host type consistently affected the risk of disease outbreak, and an association between host type and disease outbreak was still evident even after controlling for spatial differences in the location of disease outbreaks. A Bayesian spatially structured frailty model identified areas of low and high risk of downy mildew outbreak in the eastern United States. This frailty modeling framework can facilitate incorporation of spatial heterogeneity in the
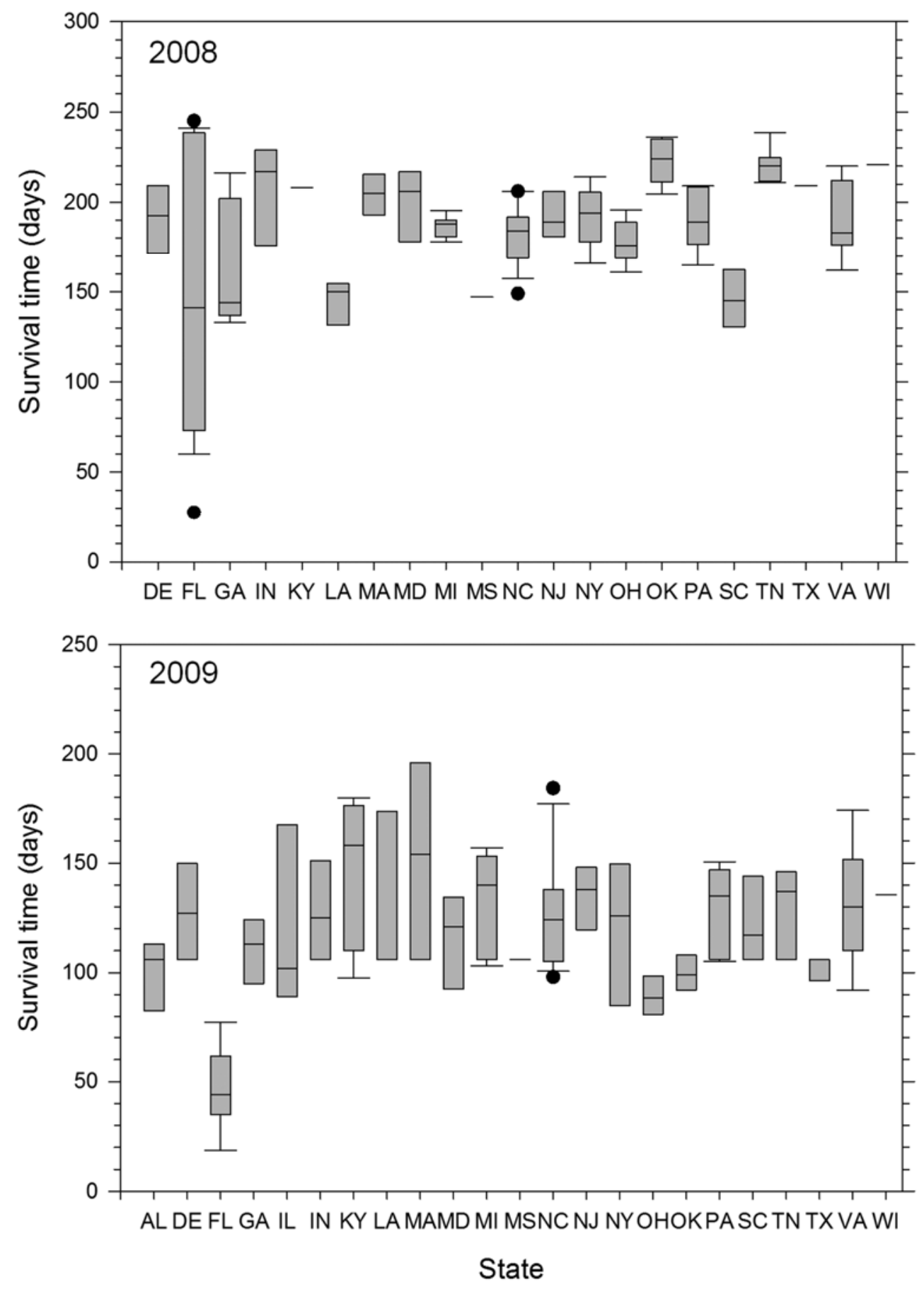

Fig. 3. Box plots showing the distribution of time to disease outbreak (survival time, days) for cucurbit downy mildew in sentinel plots in various states in the eastern United States. Vertical boxes represent the interquartile range, with the whiskers indicating the 5th and 95th percentiles, while solid lines within the boxes represent the median time to disease outbreak. 
analysis of time to disease outbreak to delineate areas with low or high risk of disease outbreak. To the best of our knowledge, this study provides the first application of Bayesian spatial frailty models in botanical epidemiology.

The spatial distribution of disease outbreaks in both years was more highly aggregated than expected from a completely random distribution of disease cases. The extent of spatial aggregation reported here is largely in agreement with the distance reported in a previous study that was based on sentinel and nonsentinel plots (24). Further, although the extent of spatial aggregation in phase II decreased when compared to that in phase I of the epidemic, separation of disease outbreaks into two phases of the epidemic did not change the clustering of disease outbreaks. Spatial pattern is an inherent property of disease dynamics and it reflects environmental forces acting on the spread and life cycle of a pathogen (29). This may explain why the spatial patterns in the two phases of the epidemic and the pattern reported in year-long spatial analysis (24) were not different. The distance over which spatial aggregation is significant corresponds to the mean distance of dispersal of the organism, plant, or disease of interest (34). When coupled with the year-long spatial analysis conducted in the previous study (24), the present study provides strong evidence to indicate that the distance over which sporangia of $P$. cubensis are expected to be transported and cause disease in cucurbit fields is $\approx 1,000 \mathrm{~km}$.

The extent and statistical significance of space-time interaction varied during the course of the epidemic. There was relatively low
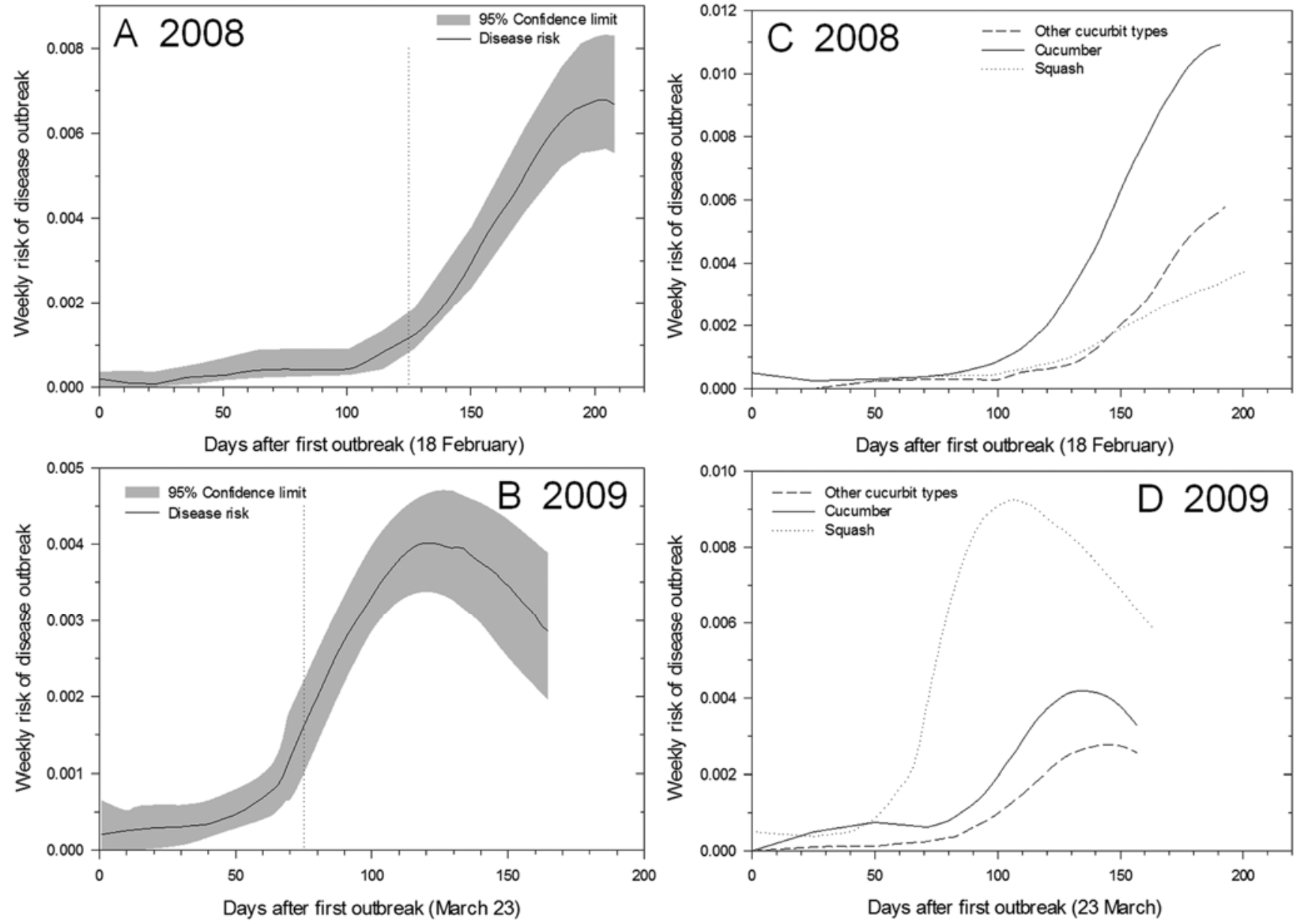

Fig. 4. Epanechnikov kernel-smoothed weekly risk of cucurbit downy mildew outbreaks in the eastern United States in A, 2008 and B, 2009. The vertical dotted line separates the two phases of the epidemic; phase I (low and sporadic disease) and phase II (high and rapid increase in disease outbreaks). The shaded area represents the $95 \%$ confidence interval around the estimates of the risk of disease outbreak. The corresponding risk of disease outbreak for different host types for the C, 2008 and D, 2009 epidemic. Other host types include cantaloupe, pumpkin, and watermelon, while squash includes both butternut and acorn squashes.

TABLE 2. Effects of cucurbit host type and regional location of outbreaks on time to disease outbreak $(T)$ in disease monitoring field plots during epidemics of cucurbit downy mildew in the eastern United States in 2008 and $2009^{\mathrm{a}}$

\begin{tabular}{|c|c|c|c|c|c|c|}
\hline \multirow[b]{2}{*}{ Variable } & \multicolumn{3}{|c|}{2008} & \multicolumn{3}{|c|}{2009} \\
\hline & $n$ & $T$ (days) mean $\pm \mathrm{SE}$ & $P>\chi^{2}$ & $n$ & $T$ (days) mean $\pm \mathrm{SE}$ & $P>\chi^{2}$ \\
\hline \multicolumn{7}{|l|}{ Host type } \\
\hline Cucumber & 118 & $196.4 \pm 4.13$ & $\ldots$ & 157 & $175.9 \pm 2.96$ & $\ldots$ \\
\hline Other & 177 & $218.6 \pm 2.73$ & $<0.0001$ & 236 & $183.2 \pm 1.80$ & $<0.0001$ \\
\hline \multicolumn{7}{|l|}{ Region } \\
\hline Southern tier & 175 & $205.6 \pm 4.25$ & & 255 & $175.3 \pm 3.07$ & \\
\hline
\end{tabular}

${ }^{a} T$ refers to time to disease outbreak after the first disease report in overwintering locations in southern Florida. Downy mildew was first reported in southern Florida on 18 February 2008 and on 23 March 2009. 
and nonsignificant spatiotemporal interaction during phase I of the epidemic. As the disease epidemic progressed, spatiotemporal interactions became more prominent and were statistically significant in phase II, as indicated by more $D_{0}(s, t)$ values $>1$. The risk of transmission as estimated by $D_{0}(s, t)$ was significant in phase II of the epidemic and was highest within $500 \mathrm{~km}$ and 1.5 months of the first disease outbreak in south Florida. This space-time risk is much narrower than that reported in the year-long spatiotemporal analysis, where $D_{0}(s, t)$ was within $600 \mathrm{~km}$ and 3 to 5 months (24). In comparison with the year-long analysis, the smaller temporal component of $D_{0}(s, t)$ in phase II of the epidemic is probably due to the shorter duration of the epidemic. The lack of statistical significance of $D_{0}(s, t)$ in phase I of the epidemic may be attributed to the sporadic and limited number of disease outbreaks as a result of limited availability of the host. Thus, during phase I of the epidemic, new infections that were occurring in the field followed a relatively random pattern. As previously observed (24), the degree and extent of contagiousness increased during the course of the epidemic. An increase in distance component of $D_{0}(s, t)=1$ contour is indicative of an increase in local-spread distances, whereas an increase in the temporal position of $D_{0}(s, t)=1$ contour suggests that infected fields are remaining infectious longer (40). The rationale that justifies the assessment of spatiotemporal pattern is that effective disease control measures aimed at reducing local spread should reduce additional risk of infection due to spatial and temporal proximity to an infected source (30). In this study, sentinel plots were not sprayed with fungicides when disease was observed. Thus, it is difficult to assess the extent to which fungicides can reduce the additional infection risk due to spatial and temporal proximity to infected sources within the region.

In botanical epidemiology, prediction of disease outbreaks at large spatial scales is often difficult. The difficulty in predicting disease outbreak at these large spatial scales is further compounded in the presence of uncertainty. As an observation, time to an outbreak of a disease has errors and the true underlying model for this event is rarely known. For any given pathosystem, these errors may be due to heterogeneity associated with the host, pathogen, or environment. This heterogeneity, which may be difficult to assess, is one of the important sources of variability in epidemiological applications. The key element here is that time to disease outbreak is different in host fields, and fields closer to each other are likely to have a similar time to disease outbreak. Consequently, disease outbreak in a given host field will sys- tematically lead to a bias in what is actually observed in any other field. The risk of disease outbreak starts to decline simply because disease has already occurred in fields close to each other but the risk of disease outbreak for an individual field may well continue to increase. Although theoretical models are able to include some of these effects in simplistic scenarios, they do not have the flexibility to accommodate the a priori uncertainty related to the dynamical assumptions of these effects. In the present study, an alternative model, a Bayesian hierarchical spatial frailty model, was formulated to predict outbreaks of downy mildew and to quantify the associated uncertainties. The model with spatial structured frailty terms provided a better fit to the data than either the unstructured frailty model or a model without frailty. Bayesian hierarchical spatial frailty CAR models have also been reported to provide good fits to time-to-event data $(3,15)$.

In this study, regions with low or high risk of disease outbreak were identified when frailties were spatially structured in a CAR model. States in the mid-Atlantic region were usually associated with high risk of downy mildew outbreak. Thus, in an effort to reduce disease spread, disease monitoring needs to be intensified in these states and control measures (e.g., fungicides) implemented once an infected field is observed in the region. This relatively large clustering of disease outbreaks is consistent with the relatively large transmission risk window identified from the space-time analysis. In addition, states with shorter estimated

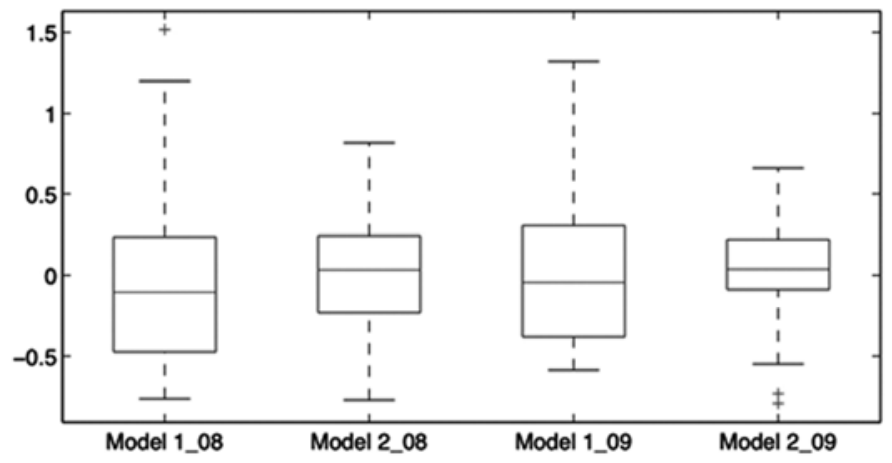

Fig. 5. Box-whisker plots showing the distribution of posterior median frailties for a model with spatially unstructured frailty (model 1) and a conditional autoregressive model with spatially structured frailty (model 2) for time to downy mildew outbreak in the 2008 (08) and 2009 (09) disease epidemics in the eastern United States.

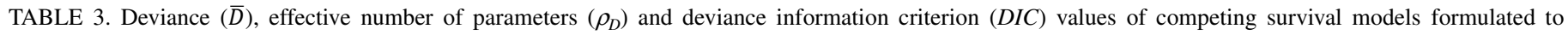
determine the risk of outbreaks of cucurbit downy mildew in the eastern United States in 2008 and $2009^{a}$

\begin{tabular}{|c|c|c|c|c|c|c|c|c|}
\hline \multirow[b]{2}{*}{ Model } & \multicolumn{4}{|c|}{2008} & \multicolumn{4}{|c|}{2009} \\
\hline & $\bar{D}$ & $\rho_{D}$ & $D I C$ & $\Delta D I C$ & $\bar{D}$ & $\rho_{D}$ & $D I C$ & $\Delta D I C$ \\
\hline No frailty & $2,541.27$ & 4.07 & $2,537.20$ & 23.41 & $2,432.16$ & 3.98 & $2,428.18$ & 18.03 \\
\hline Unstructured frailty & $2,537.47$ & 16.84 & $2,520.63$ & 6.84 & $2,431.41$ & 14.54 & $2,416.87$ & 6.72 \\
\hline Spatially structured frailty & $2,529.43$ & 15.64 & $2,513.79$ & $\ldots$ & $2,424.30$ & 14.15 & $2,410.15$ & $\ldots$ \\
\hline
\end{tabular}

a $\triangle D I C=D I C$ ("best" model) - DIC, where the spatially structured frailty model is considered as the "best" model (i.e., smallest $D I C$ ).

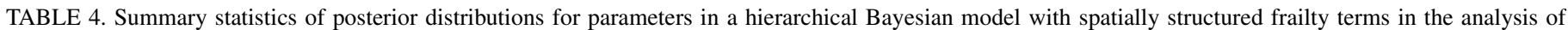
outbreaks of cucurbit downy mildew in the eastern United States in 2008 and 2009

\begin{tabular}{|c|c|c|c|c|c|c|}
\hline \multirow[b]{2}{*}{ Parameter $^{\mathrm{a}}$} & \multicolumn{3}{|c|}{2008} & \multicolumn{3}{|c|}{2009} \\
\hline & $2.5 \%$ & $50 \%$ & $97.5 \%$ & $2.5 \%$ & $50 \%$ & $97.5 \%$ \\
\hline Intercept $\left(\beta_{0}\right)$ & -23.280 & -20.596 & -18.168 & -13.663 & -12.058 & -10.573 \\
\hline Host $(=1)$ & 0.453 & 0.778 & 1.096 & 0.027 & 0.434 & 0.852 \\
\hline Host $(=2)$ & -0.675 & -0.278 & 0.101 & 0.852 & 1.206 & 1.577 \\
\hline$\rho$ & 3.161 & 3.604 & 4.092 & 1.693 & 1.970 & 2.271 \\
\hline$\lambda$ & 0.772 & 2.408 & 6.885 & 1.135 & 3.430 & 7.500 \\
\hline
\end{tabular}

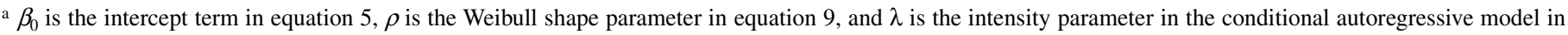
equation 12. The intercept term is included in the model purely as a computational issue to facilitate the convergence of the procedure (35) and does not affect the final model because it is absorbed in the baseline hazard. Host $=1$ and 2 represent the host groups cucumber and squash, respectively. 
times to disease outbreak corresponded closely with clusters identified by the CAR model. Host type significantly affected time to disease outbreak even after accounting for spatial differences in the location of disease outbreaks. The risk of disease outbreak due to host type differed between the 2 years, with the risk being higher for cucumber and squash in 2008 and 2009, respectively, than for other cucurbit species. When all factors are held constant, cucumber is more susceptible to downy mildew than other cucurbits and this may explain the disease risk for cucumber in 2008. However, cucurbits also exhibit a high level of host-pathogen interaction (23). Thus, it is highly likely that the pathogen strain present in 2009 may have been more virulent on squash than cucumber, resulting in a high disease risk for squash. This dynamic shift in pathogen virulence on different cucurbit
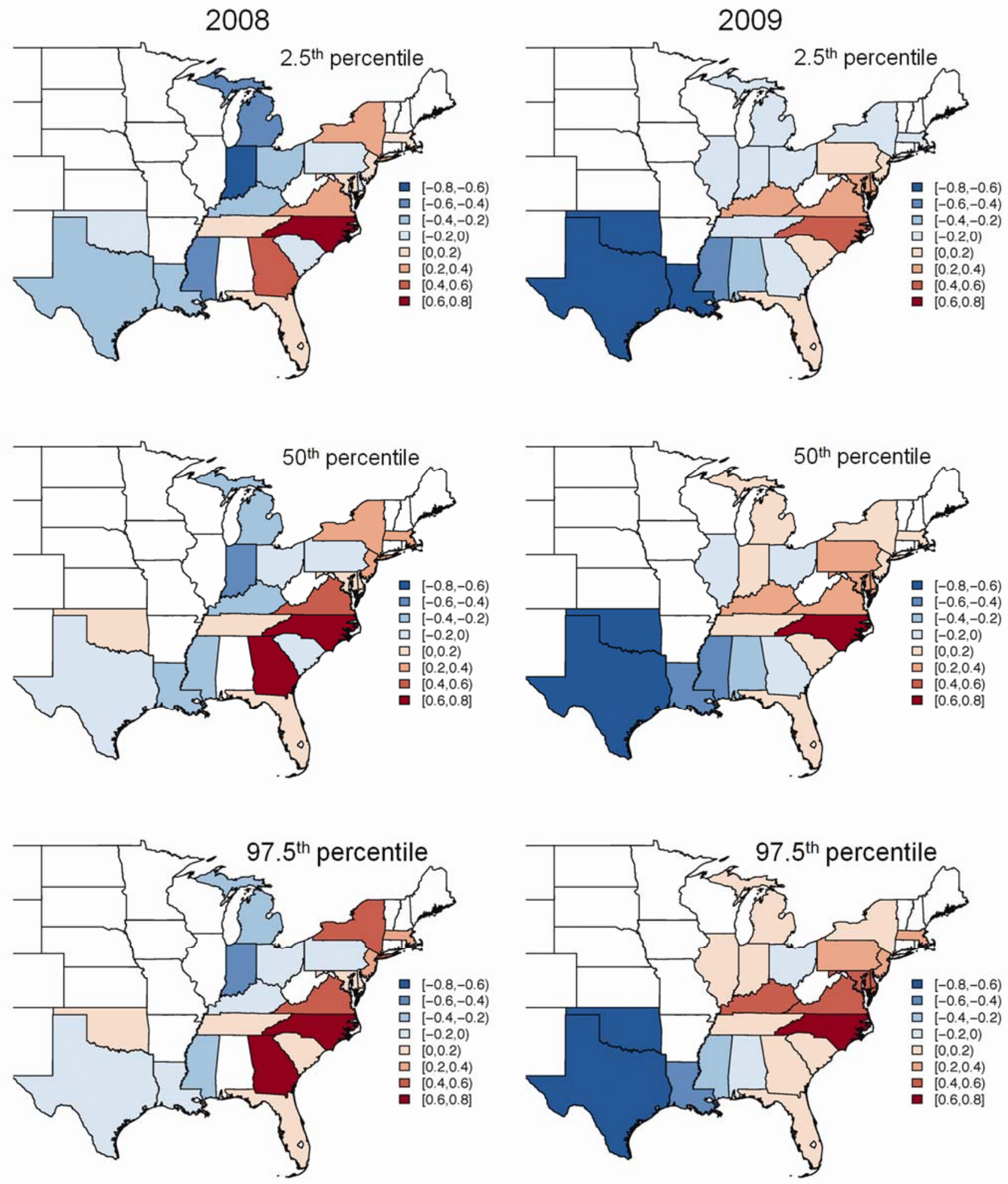

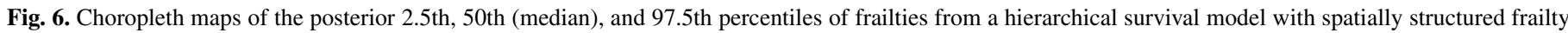

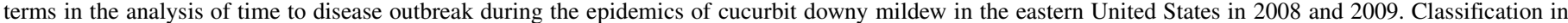

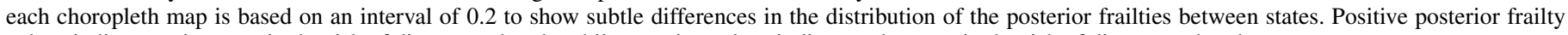
values indicate an increase in the risk of disease outbreak, while negative values indicate a decrease in the risk of disease outbreak. 
host types is supported by a recent study on $P$. cubensis conducted in the Czech Republic (22).

The possibility of ecological bias (17), where the same stratum effect is assumed to apply to all fields within a stratum, has been indicated as a possible limiting factor with the CAR approach. An alternative approach was suggested where correlations are modeled as a function of distance between point locations rather than by a lattice neighborhood structure. Henderson et al. (17) compared a stratum-level analysis with a point-level approach to investigate the spatial variation in survival of leukemia patients. Although the point-level approach produced more dispersed parameter estimates than the stratum-level analysis, the two models predicted a very similar frailty surface. Further, the 0 to 1 adjacency weighting adopted here implicitly determined a precision matrix that does not correspond to a proper covariance structure (37). This limitation can be addressed based on an approach that uses weights that satisfy certain conditions. For example, Conlon and Waller (11) investigated the use of a covariance specification based on distance and plotted the induced CAR weights versus distance to calibrate these two approaches. However, the calibration was difficult because it demanded inversion of a typical covariance structure at each iteration step, and the approach forfeited the computational simplicity of the weight-specification approach (4). Given that smoothed frailty maps were similar under the two procedures for comparable prior specifications (11), the use of a computationally simpler weight-based CAR approach provides an adequate analysis of the downy mildew data. Henderson et al. (17) developed a multivariate gamma frailty model using exact location of leukemia patients in the United Kingdom. Although this gamma frailty modeling approach is time consuming, it consistently estimated several parameters for fine-scale treatment of the data. We are currently investigating this approach for fine-scale spatial analysis of downy mildew outbreaks.

In summary, spatial heterogeneity is associated with outbreak of many plant diseases at different spatial scales. Analysis of disease outbreaks using classical survival analysis techniques assumes fixed-effects approaches, and this can generate biased estimates of the risk of disease outbreak. One reason for the lack of embrace of spatial heterogeneity in analysis of outbreaks of plant diseases has been that of computational necessity. We demonstrated that a Bayesian hierarchical spatially structured frailty model provided good predictions of the risk of downy mildew outbreak in the eastern United States. A plausible question might be whether the methodology presented here is worth the additional effort required for its implementation. First, for realistic estimates of prediction error, one must account for uncertainties in data and model. Traditional approaches to this problem simply do not account for the uncertainty in both data and model. Further, the Bayesian frailty modeling approach that accommodates uncertainties in the parameters also provided a richer understanding of the process. For example, we have, for the first time, some sense of the uncertainty in the risk of downy mildew outbreaks in the eastern United States which has not been available from traditional approaches. Thus, although the posterior distribution of median frailties indicates a high risk of downy mildew outbreak in the mid-Atlantic region, there is sufficient uncertainty in these estimates to question that interpretation. The methodology presented here can easily be extended to other plant diseases such as soybean rust and wheat stem rust. The United States Department of Agriculture routinely monitors outbreaks of invasive plant diseases in the country and provides policy advice on the likelihood of the incursion of invasive plant pathogens such as the new strain of stem rust into the United States. Accounting for spatial heterogeneity will ensure that risk estimates are not biased due to inadequate control of factors that are spatially correlated with individual disease outbreaks.

\section{ACKNOWLEDGMENTS}

This research was supported, in part, by a grant from the United States Department of Agriculture Pest Information Platform for Extension and Education (PIPE) program. E. L. Kang was partially supported by the Faculty Research Grant Program from the University Research Council and the Charles Phelps Taft Research Center at University of Cincinnati. We thank the collaborators of the Cucurbit Downy Mildew ipmPIPE project for monitoring and reporting disease outbreaks; L. Madden (Ohio State University), H. Scherm (University of Georgia), and F. Chen (SAS Institute) for some useful discussions; and the two anonymous reviewers for their constructive comments on the manuscript.

\section{LITERATURE CITED}

1. Allison, P. D. 2010. Survival Analysis Using the SAS System. A Practical Guide. SAS Institute, Cary, NC.

2. Aylor, D. E. 2003. Spread of plant disease on a continental scale: Role of aerial dispersal of pathogens. Ecology 84:1989-1997.

3. Banerjee, S., and Carlin, B. P. 2003. Semiparametric spatio-temporal frailty modeling. Environmetrics 14:523-535.

4. Banerjee, S., Carlin, B. P., and Gelfand, A. E. 2004. Hierarchical Modeling and Analysis for Spatial Data. Chapman and Hall/CRC Press, Boca Raton, FL.

5. Banerjee, S., and Dey, D. K. 2005. Semiparametric proportional odds models for spatially correlated survival data. Lifetime Data Anal. 11:175191.

6. Banerjee, S., Wall, M. M., and Carlin, B. P. 2003. Frailty modeling for spatially correlated survival data, with application to infant mortality in Minnesota. Biostatistics 4:123-142.

7. Bernardinelli, L., and Montomoli, C. 1992. Empirical Bayes versus fully Bayesian analysis of geographical variation in disease risk. Stat. Med. 11:983-1007.

8. Besag, J., York, J., and Mollié, A. 1991. Bayesian image restoration, with two applications in spatial statistics. Ann. Inst. Stat. Math. 43:1-59.

9. Carlin, B. P., and Hodges, J. S. 1999. Hierarchical proportional hazards regression models for highly stratified data. Biometrics 55:1162-1170.

10. Carlin, B. P., and Louis, T. A. 2000. Bayes and Empirical Bayes Methods for Data Analysis, 2nd Edition. Chapman and Hall/CRC Press, Boca Raton, FL.

11. Conlon, E. M., and Waller, L. A. 1999. Flexible spatial hierarchical models for mapping disease rates. Pages 82-87 in: Proc. A.S.A. Sect. Stat. Environ. American Statistical Association, Washington, DC.

12. Cox, D. R. 1972. Regression models and life tables (with discussion). J. R. Stat. Soc. B 34:187-220.

13. Cox, D. R., and Oakes, D. 1984. Analysis of Survival Data. Chapman and Hall, London.

14. Diggle, P. J., Chetwynd, A. G., Haggkvist, R., and Morris, S. E. 1995. Second-order analysis of space-time clustering. Stat. Methods Med. Res. 4:124-136.

15. Diva, U., Dey, D. K., and Banerjee, S. 2008. Parametric models for spatially correlated survival data for individuals with multiple cancers. Stat. Med. 27:2127-2144.

16. Gelfand, A. E., and Smith, A. F. M. 1990. Sampling-based approaches to calculating marginal densities. J. Am. Stat. Assoc. 85:398-409.

17. Henderson, R., Shimakura, S., and Gorst, D. 2002. Modeling spatial variation in leukemia survival data. J. Am. Stat. Assoc. 97:965-972.

18. Holmes, G., Wehner, T., and Thornton, A. 2006. An old enemy reemerges: Downy mildew rears its ugly head on cucumber, impacting growers up and down the Eastern U.S. Am. Veg. Grow. February:14-15.

19. Hougaard, P. 1995. Frailty models for survival analysis. Lifetime Data Anal. 1:255-273.

20. Lawson, A. B. 2009. Bayesian Disease Mapping: Hierarchical Modeling in Spatial Epidemiology. Chapman and Hall/CRC Press, Boca Raton, FL.

21. Lebeda, A., and Cohen, Y. 2011. Cucurbit downy mildew (Pseudoperonospora cubensis)-biology, ecology, epidemiology, host-pathogen interaction and control. Eur. J. Plant Pathol. 129:157-192.

22. Lebeda, A., Pavelková, J., Sedláková, B., and Urban, J. 2012. Structure and temporal shift of pathogenic variation of Czech Pseudoperonospora cubensis populations. Plant Pathol. Online publication. doi:10.1111/ j.1365-3059.2012.02642.x

23. Lebeda, A., Widrlechner, M. P., and Urban, J. 2006. Individual and population aspects of interactions between cucurbits and Pseudoperonospora cubensis: Pathotypes and races. Pages 453-467 in: Proceedings of Cucurbitaceae. G. J. Holmes, ed. Universal Press, Asheville, NC.

24. Ojiambo, P. S., and Holmes, G. J. 2011. Spatiotemporal spread of cucurbit downy mildew in the eastern United States. Phytopathology 101:451-461. 
25. Ojiambo, P. S., Holmes, G. J., Britton, W., Keever, T., Adams, M. L., Babadoost, M., Bost, S. C., Boyles, R., Brooks, M., Damicone, J., Draper, M. A., Egel, D. S., Everts, K. L., Ferrin, D. M., Gevens, A. J., Gugino, B. K., Hausbeck, M. K., Ingram, D. M., Isakeit, T., Keinath, A. P., Koike, S. T., Langston, D., McGrath, M. T., Miller, S. A., Mulrooney, R., Rideout, S., Roddy, E., Seebold, K. W., Sikora, E. J., Thornton, A., Wick, R. L., Wyenandt, C. A., and Zhang, S. 2011. Cucurbit downy mildew ipmPIPE: a next generation web-based interactive tool for disease management and extension outreach. Online. Plant Health Progress. Online publication. doi:10.1094/PHP-2011-0411-01-RV

26. Ojiambo, P. S., and Scherm, H. 2005. Survival analysis of time to abscission of blueberry leaves affected by Septoria leaf spot. Phytopathology 95:108-113.

27. Real, L. A., and Biek, R. 2007. Spatial dynamics and genetics of infectious diseases on heterogeneous landscapes. J. R. Soc. Interf. 4:935948.

28. Ripley, B. D. 1981. Spatial Statistics. John Wiley \& Sons, Inc., Hoboken, NJ.

29. Ristaino, J. B., and Gumpertz, M. 2000. New frontiers in the study of dispersal and spatial scales of epidemics by species in the genus Phytophthora. Annu. Rev. Phytopathol. 38:541-576.

30. Sanchez, J., Stryhn, H., Flensburg, M., Ersbøll, A. K., and Dohoo, I. 2005. Temporal and spatial analysis of the 1999 outbreak of acute clinical infectious bursal disease in broiler flocks in Denmark. Prev. Vet. Med. 71:209-223.
31. Scherm, H., and Ojiambo, P. S. 2004. Applications of survival analysis in botanical epidemiology. Phytopathology 94:1022-1026.

32. Spiegelhalter, D. J., Best, N. G., and Carlin, B. P. 2002. Bayesian measures of model complexity and fit. J. R. Stat. Soc. B 64:583-639.

33. Spiegelhalter, D. J., Thomas, A., Best, N. G., and Lunn, D. 2003. WinBUGS User Manual, Version 1.4. MRC Biostatistics Unit, Institute of Public Health and Department of Epidemiology and Public Health, Imperial College School of Medicine, UK. http://www.mrc-bsu.cam. ac.uk/bugs

34. Suzuki, R. O., Suzuki, J., and Kachi, N. 2005. Change in spatial distribution of a biennial plant between growth stages and generation in a patchy habitat. Ann. Bot. 96:1009-1017.

35. Tibshirani, T. 1997. The lasso method for variable selection in the cox model. Stat. Med. 16:385-395.

36. Van der Plank, J. E. 1963. Plant Diseases: Epidemics and Control. Academic Press, Inc., New York.

37. Wall, M. M. 2004. A close look at the spatial structure implied by the CAR and SAR models. J. Stat. Plan. Infer. 121:311-324.

38. Wiegand, T., and Moloney, K. A. 2004. Rings, circles and null-models for point pattern analysis in ecology. Oikos 104:209-229.

39. Wikle, C. K. 2003. Hierarchical Bayesian models for predicting the spread of ecological processes. Ecology 84:1382-1394.

40. Wilesmith, J. W., Stevenson, M. A., King, C. B., and Morris, R. S. 2003. Spatio-temporal epidemiology of foot-and-mouth disease in two counties of Great Britain in 2001. Prev. Vet. Med. 61:157-170. 